\title{
EHMTI-0052. Efficacy of early vs. late use of frovatriptan combined with dexketoprofen vs. frovatriptan alone in the acute treatment of migraine attacks with or without aura
}

\author{
G Allais $^{1 *}$, V Tullo ${ }^{2}$, P Cortelli ${ }^{3}$, P Barbanti ${ }^{4}$, F Valguarnera ${ }^{5}, G$ Sette $^{6}$, F D'Onofrio ${ }^{7}$, M Curone $^{2}$, G Reggiardo $^{8}$,
} S Omboni ${ }^{9}$, F Frediani $^{10}$, G Bussone $^{2}$, C Benedetto $^{1}$

From 4th European Headache and Migraine Trust International Congress: EHMTIC 2014

Copenhagen, Denmark. 18-21 September 2014

\begin{abstract}
Aims
To compare frovatriptan $2.5 \mathrm{mg}$ plus dexketoprofen 25 or $37.5 \mathrm{mg}$ (FroDex 25 and FroDex 37.5) vs. frovatriptan 2.5 $\mathrm{mg}$ (Frova) in the acute treatment of migraine attacks in a post-hoc analysis of a double-blind, randomized, parallelgroup study. Patients who took the drug within $30 \mathrm{~min}$ from the onset of pain (early use, EU) or after (late use, LU) were analyzed.
\end{abstract}

\section{Methods}

314 migraineurs with or without aura treated at least one attack with Frova, FroDex 25 or FroDex 37.5. Traditional migraine endpoints were compared across study drugs in the 279 patients of the full analysis set according to $\mathrm{EU}(\mathrm{n}=172)$ or $\mathrm{LU}(\mathrm{n}=107)$.

\section{Results}

Pain free (PF) at 2-hours in EU was 33\% with Frova, 50\% with FroDex 25 and 51\% with FroDex 37.5 (p = NS), while in LU was $22 \%, 51 \%$ and $50 \%$ ( $<<0.05$ combinations vs. monotherapy), respectively. PF at 4-hours was $54 \%$ for EU and $34 \%$ for LU of Frova, 71\% and 57\% with FroDex 25 and $74 \%$ and $68 \%$ with FroDex 37.5 (p $<0.05$ for EU and $\mathrm{p}<0.01$ for LU vs. Frova). Sustained pain free at 24-hours was $26 \%$ under Frova, $43 \%$ under FroDex 25 and $40 \%$ under FroDex 37.5 ( $\mathrm{p}=\mathrm{NS}$ ) in EU, while it was 19\% under Frova, 43\% under FroDex 25 and 45\% under FroDex 37.5 ( $p<0.05$ FroDex 25 and FroDex 37.5 vs. Frova) in LU. Risk of relapse at 48-hours was similar $(\mathrm{p}=\mathrm{NS})$ among study groups (Frova: 25\%, FroDex 25: 21\%, and FroDex 37.5: 37\%) for both EU and LU (14\%, $42 \%$ and $32 \%)$.

\section{Conclusions}

FroDex was found to be more effective than Frova taken either early or late.

\section{Authors' details}

'Department of Gynecology and Obstetrics, Women's Headache Center, Torino, Italy. ${ }^{2}$ Clinical Neuroscience, National Neurological Institute Carlo Besta, Milano, Italy. ${ }^{3}$ Neurological Science, University of Bologna, Bologna, Italy. ${ }^{4}$ Headache and Pain Unit Department of Neurological Motor and Sensorial Sciences, IRCCS San Raffaele Pisana, Roma, Italy. ${ }^{5}$ Department Head-Neck, Sestri Ponente Hospital, Genova, Italy. ${ }^{6}$ Neurological Headache Centre, Sant'Andrea Hospital, Roma, Italy. ${ }^{7}$ Neurologic Unit, San Giuseppe Moscati Hospital, Avellino, Italy. ${ }^{8}$ Biometric Unit, Mediservice, Milano, Italy. ${ }^{9}$ Clinical Research Unit, Italian Institute of Telemedicine, Solbiate Arno (Varese), Italy. ${ }^{10}$ Headache Center, Ospedale San Carlo Borromeo, Milano, Italy.

Published: 18 September 2014

\section{doi:10.1186/1129-2377-15-S1-G3}

Cite this article as: Allais et al.: EHMTI-0052. Efficacy of early vs. late use of frovatriptan combined with dexketoprofen vs. frovatriptan alone in the acute treatment of migraine attacks with or without aura. The Journal of Headache and Pain 2014 15(Suppl 1):G3. 